\title{
Progress in quantitative analysis of plant hormones
}

\author{
FU JiHong ${ }^{1,2}$, SUN XiaoHong ${ }^{2}$, WANG JiDe ${ }^{1}$, CHU JinFang $^{2 *} \&$ YAN CunYu $^{2 *}$ \\ ${ }^{1}$ College of Chemistry and Chemical Engineering, Xinjiang University, Urumqi 830046, China; \\ ${ }^{2}$ National Centre for Plant Gene Research (Beijing), Institute of Genetics and Developmental Biology, Chinese Academy of Sciences, Beijing \\ 100101, China
}

Received October 27, 2010; accepted November 11, 2010

\begin{abstract}
Plant hormones are small molecular natural products that regulate all plant developmental processes at low concentrations. Quantitative analysis of plant hormones is increasingly important for in-depth study of their biosynthesis, transport, metabolism and molecular regulatory mechanisms. Although plant hormone analysis remains a bottleneck in plant scientific research owing to the trace concentrations and complex components in plant crude extracts, much progress has been achieved in the development of extraction, purification and detection techniques in recent years. Solid phase extraction and chromatography/mass spectrometry have been applied widely for purification and quantitative analysis of plant hormones owing to their high selectivity and sensitivity. Purification methods such as liquid partition and immunoaffinity chromatography, and detection methods including immunoassay and electrochemical analysis, are employed. The advantages and disadvantages of these methods are discussed. In situ, real-time and multi-plant hormone profiling will comprise mainstream techniques for quantitative analyses in future studies on the regulatory mechanisms and crosstalk of plant hormones.
\end{abstract}

plant hormones, quantitative analysis, solid phase extraction, liquid chromatography, gas chromatography, mass spectrometry

Citation: $\quad$ Fu J H, Sun X H, Wang J D, et al. Progress in quantitative analysis of plant hormones. Chinese Sci Bull, 2011, 56: 355-366, doi: 10.1007/s11434-0104243-8

Plant hormones are naturally occurring secondary metabolites that play key roles in the whole life cycle of plants, including cell division, enlargement and differentiation, organ development, seed dormancy and germination, leaf and organ senescence, and abscission [1]. Currently recognized plant hormones include ethylene, auxin, abscisic acid, gibberellins, cytokinins, jasmonates, brassinosteroids and salicylates (Table 1). Some additional special secondary metabolites, such as nitric oxide (NO) and the newly discovered strigolactones, are also identified as plant hormones owing to their similar modes of action. A number of inducible defense mechanisms are involved in plant responses to both biotic and abiotic stresses such as pathogen attack, lesions resulting from insect feeding or other damage, drought and salinity [2-4], and most importantly, these

\footnotetext{
*Corresponding authors (email: cunyuyan@genetics.ac.cn; jfchu@genetics.ac.cn)
}

defense responses are all triggered by plant hormones.

A plant hormone, acting as a plant-development controller or a plant-environment mediator, can influence crop yield directly or indirectly, therefore research into plant hormone physiology has become an important target for agriculture development. However, it is noteworthy that endogenous level of each plant hormone is very low [1], and quantitative analysis of these compounds is still extremely difficult for both biologists and analytical chemists. It is a challenge for researchers to enrich these low-level natural compounds from plant extracts in which large amounts of interfering substances are present. In addition, the limited quantities of plant tissue available for hormone quantification is another challenge. Consequently, the analytic methods must be extremely selective and sensitive. The quantitative analysis of plant hormones typically includes the following key points: (i) the plant materials must 
Table 1 Structures and names of representatives of the major plant hormones

\begin{tabular}{|c|c|c|}
\hline \multirow{2}{*}{ Category } & \multicolumn{2}{|c|}{ Representative of each major plant hormone } \\
\hline & Structure & Name \\
\hline Auxins & & Indole-3-acetic acid (IAA) \\
\hline Abscisic acid (ABA) & & S-(+)-abscisic acid (S-ABA) \\
\hline Jasmonates (JAs) & & (-)-Jasmonic acid (-)-JA \\
\hline Gibberellins (GAs) & & Gibberellin $\mathrm{A}_{1}\left(\mathrm{GA}_{1}\right)$ \\
\hline Salicylates (SAs) & & Salicylic acid (SA) \\
\hline Cytokinins (CTKs) & & trans-Zeatin (Z) \\
\hline Ethylene & $\mathrm{H}_{2} \mathrm{C}=\mathrm{CH}_{2}$ & Ethylene \\
\hline Strigolactones (SLs) & & 2'-epi-5-Deoxylstrigol (epi-5DS) \\
\hline Brassinosteroids (BRs) & & Brassinolide (BR) \\
\hline
\end{tabular}

be maintained in an intact state during harvesting to avoid affecting the subsequent analysis; (ii) the proper solvent should be chosen for efficient extraction, and the solvent must make contact with the target analytes; (iii) the method for purification and enrichment of plant extracts should be optimized; and (iv) development of sensitive and accurate analytical methods to determine hormone levels in the plant tissues is necessary. In recent years, significant technical advances in plant hormone extraction and detection have shed light on hormone metabolism, signal transduction and plant hormone crosstalk networks. In 2007, the National Natural Science Foundation of China initiated a major research project on the topic of plant hormones, and one of the supported area is related to the quantitative detection of plant hormones, which is still the bottleneck for plant hormone physiology research. The aim of this review is to summarize these recent advances in the methods for quantification of plant hormones and to highlight key areas of future investigation.

\section{Extraction and purification}

As a special subset of plant secondary metabolites, plant hormones occur at very low concentrations of $0.1-50 \mathrm{ng} \mathrm{g}^{-1}$ 
fresh weight inside the plant, which are about one tenthousandth or even lower than the concentration of other types of plant secondary metabolites such as flavonoids [5]. In addition, many plant hormones coexist with other endogenous organic compounds in plant extracts, which may interfere with the final assay of the plant hormones. Thus it is important to design an extraction and purification strategy that can remove the interfering substances and enrich the target compounds from the complex plant extracts.

\subsection{Extraction}

Sample preparation is a key procedure in plant hormone analysis. First, plant materials is kept cold to avoid enzymatic induction of metabolic change or chemical degradation of the target compounds, and then the fresh or freeze-dried materials is ground into fine powder in liquid nitrogen. Second, an appropriate solvent must be selected to extract the compounds efficiently from the targeted tissues.

At present, solvent extraction is still the most widely used method for plant hormone extraction. Many procedures for extraction have been developed and diverse solvents such as methanol, methanol/water mixture, acetone, acetone/water, propanol, propanol/water, and neutral or acid buffer are broadly used [6-13]. The polarity of the extraction solvent should match closely that of the target compound, and thereby the ratio of organic solvent to water is defined according to the polarity of hormones. Non-polar solvents such as ether are rarely used to extract plant hormones. Of these organic solvents, methanol has become the preferred solvent and is widely used for extraction of plant hormones. Its small size and low molecular weight allow it to efficiently penetrate into plant cells during extraction [14-23]. Another notable problem is that some plant hormones are labile during the extraction process. Auxins, for example, are readily oxidized or degraded when exposed to light, oxygen and high temperature. To protect against their degradation, addition of antioxidants during the extraction process is ne- cessary. As well as the external environment, many hormones, such as cytokinins, also can be degraded by endogenous enzymes. In this case, plant materials should be immediately frozen or extracted with a suitable solvent to prevent any enzymatic degradation. Attention should be paid to phosphatases, which can catalyze the hydrolysis of cytokinin riboside phosphates during extraction even with $80 \%$ methanol solvent. Bieleski [24] developed a solvent consisting of $\mathrm{MeOH}: \mathrm{CHCl}_{3}$ : $\mathrm{H}-\mathrm{COOH}: \mathrm{H}_{2} \mathrm{O}(12: 5: 1: 2, \mathrm{v} / \mathrm{v} / \mathrm{v} / \mathrm{v})$ that was found to inactivate phosphatases in a variety of plant materials. However, the presence of $\mathrm{CHCl}_{3}$ in Bieleski's solvent will extract lipophilic components from the plant tissue, which makes subsequent purification of hormones more complicated [25,26]. A modified Bieleski's solvent (MeOH:HCOOH: $\mathrm{H}_{2} \mathrm{O}=15: 1: 4$, v/v/v) was developed by adjusting the organic solvents and their ratio, and has been used widely for extraction of cytokinins and gibberellins [27,28]. Although the modified Bieleski's solvent does not extract lipophilic components, it can still release free cytokinins from cytokinin nucleosides, that affects the accuracy and reliability of the determination of endogenous cytokinins.

The choice of extract solvent depends not only on the physicochemical properties of target analytes but also on the types of plant materials. The extract solvent should vary with the extraction conditions and the forms of the hormones present in the plant sample. Ideal solvents should extract the hormone efficiently and avoid extracting additional, interfering substances from the plant tissues.

\subsection{Purification}

Because hormones are present in plant tissues at very low concentrations, and their determination can be hindered by hundreds of other abundant primary and secondary metabolites, the purification and enrichment of hormones is very crucial for the final assay. For this purpose, different methods for further purification and enrichment of the crude extracts should be carried out. An increasing number of methods and techniques have been adapted for sample purification.

(i) Liquid-liquid extraction. Liquid-liquid extraction, also known as solvent extraction and liquid partition, is a method to separate compounds based on their relative solubility in 2 different immiscible liquids, usually with a water and organic solvent system. Ideal partition results can be obtained by liquid-liquid extraction for some relatively high-level plant hormones. Many organic solvents, such as ethyl acetate, dichloromethane and diethyl ether, are used to purify plant hormones according to the physicochemical properties of the target hormones [7,9,15]. However, traditional liquid extraction involves many extraction steps and is a time- and solvent-consuming work. Additionally, it should be noted that liquid-liquid extraction can reduce the extraction efficiency of water-soluble plant hormones because of serious emulsification. All these disadvantages have seen this method used decreasingly in laboratory studies.

Recently, a new liquid extraction method was developed by improving the traditional technique. The new method was used to extract and concentrate four acidic plant hormones (IAA, ABA, SA and JA) simultaneously in natural coconut juice through a hollow fiber-based liquid-liquidliquid microextraction [29]. Although it is a fast and low solvent-consuming method, the complicated organic solvent selection criteria and experimental design still limit its application. In the liquid-liquid-liquid phase microextraction mode, the analyte is extracted from an aqueous solution (donor phase) into another $\mathrm{pH}$-different aqueous phase (acceptor phase) through an organic solvent (organic phase). The suitable solvent should be immiscible with water to avoid dissolution during the extraction and has a similar polarity to the hollow fiber. The solubility of the analytes in the organic solvent should be higher than that in the first aqueous solution, and lower than that in the 2 nd aqueous 
solution. Taken together, the liquid-liquid and improved liquid-liquid-liquid methods only partly solve the problems of plant hormone purification and enrichment, and their efficiencies are far from meeting the researcher's requirements for fast, convenient and high-throughput sample analysis.

(ii) Solid-phase extraction. Solid-phase extraction (SPE) differs from the liquid-liquid method in that a SPE column is used to separate the target compounds from other compounds dissolved or suspended in the same liquid mixture according to differences in the physicochemical properties of the compounds. The aim of removing the interference substances and enriching the target hormones from the samples can be achieved by trapping the target compounds on the SPE column and eluting the interference compounds or vice versa. The affinity of the target or interfering compounds on the SPE column is the only criteria for the researcher to determine which handling process is better for their experiment. Compared to the liquid-liquid extraction method, SPE is a time-saving method that consumes fewer solvents during sample processing and also potentially affords the possibility of high-throughput device which will be used in the field of plant hormone analysis. Many types of commercial SPE columns can be applied for the extraction and enrichment of different kinds of plant hormones. To increase the efficiency of purification, the solid particulate medium and sample processing must be investigated according to the different physicochemical properties of the target plant hormones.

Some laboratories use a High-Performance Liquid Chromatography (HPLC) fraction to enrich hormones during plant sample handling. HPLC can be regarded as a special solid-phase extraction technique and can enrich the target hormones efficiently and remove interfering compounds significantly. Currently, a gel column, $\mathrm{C}_{18}$ column and ODS column have been used for the purification of plant hormones [30-32]. Fractionation by HPLC is more accurate and reliable than that of other methods. HPLC, however, has disadvantages such as a longer running time, consumes more of organic solvents and is more expensive compared to SPE methods. The method has been seldom used for purification of hormones except those occurring at much lower concentrations such as gibberellins [33].

Solid phase extraction provides more streamlined sample processing compared with HPLC fractionation. Owing to the faster sample preparation and better reproducibility, SPE is used widely for the extraction of plant hormones. A wide range of high-quality SPE columns are available, offering a variety of separation modes for different applications. The major classes of sorbents in use are summarized in Table 2. The main separation modes used are ion-exchange [34,35], reversed-phase $\left(\mathrm{C}_{18},[16,36-40]\right.$; Oasis HLB, $\left.[8,28,41]\right)$ and mixed mode (Oasis MAX, [41]; Oasis MCX, [28,39,40]). Considering the complex interference compounds that might be present in the plant extracts, more than one kind of sorbent is used to enrich the plant hormones efficiently.
Among the sorbents, $\mathrm{C}_{18}$ is the most widely used. When the SPE column is conditioned with weak acid solution and the plant extracts' $\mathrm{pH}$ is adjusted with weaker acid, the acidic plant hormones can be kept in a neutral form and trapped on the $\mathrm{C}_{18}$ column before sample loading. After loading the sample, the target analyte is eluted with the corresponding acidic aqueous solution of methanol or ethanol (generally, the acidity is adjusted by formic acid or acetic acid). $\mathrm{C}_{18}$ is apparently extremely efficient in the removal of polar compounds and plant pigments. However, Hou et al. [42] found that non-acidified methanol solutions had stronger eluting abilities than acidified solutions because acidified methanol can keep the carboxyl-containing plant hormones in a neutral form that is much more strongly retained on the $\mathrm{C}_{18}$ column. The sample matrix, physicochemical properties of the analytes, and the nature of the bonded phase should be taken into account when choosing SPE columns. Combinations of two or more different kinds of SPE columns are often used in plant hormone analysis. Anion-exchange columns and cation-exchange columns are often used to extract acidic analytes and basic analytes, respectively [27,40,41]. During extraction, the $\mathrm{pH}$ of the sample is adjusted to ionize the target molecules so that the target molecules can be adsorbed on the oppositely charged sites of the sorbent. Subsequently, the anion or cation analytes are eluted with the corresponding acidic or basic aqueous solution of methanol. Cytokinins are amphoteric plant hormones with a $\mathrm{pKa} \approx 4$ with a nitrogen atom at the $6-\mathrm{C}$ of purine and a $\mathrm{pKa} \approx 10$ with a nitrogen atom in the imidazole ring; these properties can protonate and deprotonate the cytokinins under $\mathrm{pH}<3$ and $\mathrm{pH}>11$ solvent conditions, respectively [25]. All cytokinins can be ionized under an appropriate $\mathrm{pH}$, whereas the endogenous interference cytokinin-nucleotides cannot because they bear a phosphate group that possesses a diacid property with $\mathrm{pKa}_{1} \approx 1.0$ and $\mathrm{pKa}_{2} \approx 6.0$. Thus cytokinins can be separated and purified rapidly and efficiently from the interference background using mixed-mode SPE with both reversed-phase and ion-exchange extraction. Ge et al. [40] have developed an efficient dual-step SPE method for preconcentration and purification of cytokinins in coconut water using $\mathrm{C}_{18}$ and MAX column. A $\mathrm{C}_{18}$ column is extremely efficient for removal of lipids and some plant pigments. After preconcentration with a $\mathrm{C}_{18}$ SPE column, further sample purification can be carried out using mixed-mode cation exchanger (MCX) SPE cartridges with good recoveries. The cytokinins are separated from Oasis MCX columns using solvents containing different concentrations of methanol and $\mathrm{NH}_{4} \mathrm{OH}$. Purification of cytokinins using MCX SPE is also suitable for removal of ultraviolet (UV) absorbing contaminants combined with higher recoveries of cytokinins [27]. The cytokinin nucleotides can be eluted with $0.35 \mathrm{~mol} \mathrm{~L}^{-1} \mathrm{NH}_{4} \mathrm{OH}$; the cytokinin bases, ribosides and glucosides can be eluted with $0.35 \mathrm{~mol} \mathrm{~L}^{-1}$ $\mathrm{NH}_{4} \mathrm{OH}$ in $60 \% \mathrm{CH}_{3} \mathrm{OH}$.

It is important to obtain the $\mathrm{pKa}$ value of the target hor- 
Table 2 Solid-phase extraction (SPE) applications in plant hormone analysis

\begin{tabular}{|c|c|c|c|c|c|c|}
\hline SPE name & Classes & Principle & Character & $\mathrm{pH}$ & Analytes & References \\
\hline DEAE-cellulose & Anion-exchange & $\begin{array}{c}\text { DEAE-cellulose sorbent is diethylaminoethyl cel- } \\
\text { lulose, which provides anion-exchange } \\
\text { retention }\end{array}$ & $\begin{array}{l}\text { Anion/ } \\
\text { moderately polar }\end{array}$ & $2-9.5$ & CTKs & [34] \\
\hline SCX & Cation-exchange & $\begin{array}{l}\text { SCX is a silica-based sorbent with a chemically } \\
\text { bonded benzenesulfonic acid functional group, } \\
\text { which provides cation-exchange retention }\end{array}$ & Cation/nonpolar & $2-8$ & CTKs & {$[35]$} \\
\hline $\mathrm{C}_{18}$ & Reversed-phase & $\begin{array}{c}\text { Strongly hydrophobic silica-based } \\
\text { bonded phase }\end{array}$ & $\begin{array}{l}\text { Nonpolar/ } \\
\text { slightly polar }\end{array}$ & $2-7.5$ & $\begin{array}{l}\text { JA, ABA, IAA, } \\
\text { IBA, GA, Z, BA, } \\
\text { KT, CTKs }\end{array}$ & {$[16,36-40]$} \\
\hline Oasis HLB & Reversed-phase & $\begin{array}{l}\text { HLB sorbent is a macroporous copolymer made } \\
\text { from a balanced ratio of two monomers, the lipo- } \\
\text { philic divinylbenzene and the hydrophilic } \\
\qquad N \text {-vinylpyrrolidone }\end{array}$ & $\begin{array}{l}\text { Nonpolar/ } \\
\text { slightly polar }\end{array}$ & $1-14$ & $\begin{array}{l}\text { IAA, ABA } \\
\text { GA, CTKs }\end{array}$ & {$[8,28,41]$} \\
\hline Oasis MAX & $\begin{array}{l}\text { Mixed mode } \\
\text { reversed-phase/ } \\
\text { anion-exchange }\end{array}$ & $\begin{array}{c}\text { MAX sorbent is built upon HLB copolymer } \\
\text { containing two monomers, hydrophilic } \\
N \text {-vinylpyrrolidone and lipophilic divinylbenzene, } \\
\text { which provides both ion-exchange and } \\
\text { reversed-phase retention. The additional presence } \\
\text { of quaternary ammonium groups allows for } \\
\text { anion-exchange interactions }\end{array}$ & Anion/nonpolar & $1-14$ & $\begin{array}{l}\text { IAA, ABA, } \\
\text { CTKs }\end{array}$ & {$[41,43]$} \\
\hline Oasis MCX & $\begin{array}{l}\text { Mixed mode } \\
\text { reversed-phase/ } \\
\text { cation-exchange }\end{array}$ & $\begin{array}{c}\text { MCX sorbent is built upon HLB copolymer } \\
\text { containing two monomers, hydrophilic } \\
N \text {-vinylpyrrolidone and lipophilic divinylbenzene, } \\
\text { which provides both ion-exchange and } \\
\text { reversed-phase retention. The additional presence } \\
\text { of sulfonic groups allows for cation-exchange } \\
\text { interactions }\end{array}$ & Cation/nonpolar & $1-14$ & $\begin{array}{l}\text { IAA, ABA, GA, } \\
\text { KT, CTKs }\end{array}$ & {$[28,39,40]$} \\
\hline
\end{tabular}

mone before developing a hormone analysis method based on ion-exchange, reversed-phase and mixed modes SPE. The solution $\mathrm{pH}$ is adjusted to present analytes in a positive charge, negative charge or neutral form according to their $\mathrm{pKa}$. The acidity and proportion of organic solvents in the eluent determine their eluting power. Therefore, the protocol must be optimized to purify the plant hormones efficiently from the plant extracts.

Some volatile plant hormones or derived plant hormones can be enriched using polymeric adsorbent (Super Q), which is an easy and cheap method to gain ideal results. Jasmonic acid (JA) and salicylic acid (SA) are thought to be central components of signaling pathways to activate plant defense responses [44-47]. Methyl jasmonate (MeJA) and methyl salicylate (MeSA) volatilize at a relatively low temperature, which enables these compounds to be trapped on SuperQ filter material. Engelberth et al. [13] developed such a method with only one step based on the collection of the volatile derived compounds on Super Q. Engelberth et al. used citric acid in water/acetone $(30: 70, \mathrm{v} / \mathrm{v})$ to extract JA and SA. They selected acetone/citric acid/water as the extraction solvent rather than methanol/water mainly because some components would be methylated under the methanol/water extraction condition, which will cause serious loss of components during evaporation of the solvent. Additionally, the boiling point $\left(56^{\circ} \mathrm{C}\right)$ of acetone is lower than that of methanol $\left(63.8^{\circ} \mathrm{C}\right)$ and thus it is easier to be evaporated. The trapped MeJA and MeSA were eluted with dichloromethane for further quantification with gas chromatography
(GC)/mass spectrometry (MS). This method omits further purification steps with high recovery, and is convenient and inexpensive for quantification of some plant hormones.

Solid-phase microextraction (SPME) is a recently developed SPE technique in which the extraction, concentration, desorption and injection of analytes is realized simultaneously using a syringe-like injection device. The method has already been used for plant hormone purification [48]. The analytes must have properties similar to those of the coating material to be transported from the bulk matrix to the fiber coating and reach equilibrium quickly. The SPME extraction efficiencies are strongly influenced by fiber type, $\mathrm{pH}$ value, salinity, temperature and extraction time. Although SPME has been applied widely in combination with GC, application of SPME-HPLC is still limited because of the small number of commercially available fiber coatings. In the SPME-HPLC system, the SPME fibre-absorbed target analytes are transferred to the HPLC injection port and eluted in the mobile phase. The coating materials must have sufficient tolerance in the mobile phase and maintain stability in acetonitrile, methanol and other organic solvents.

Solid phase extraction technology greatly simplifies plant hormone purification and provides high-throughput capability. It has become a proven and general sample processing technology. Different kinds of sorbents and many sizes of SPE columns can be chosen to meet the requirements of a variety of sample pretreatments. It is a growing technological area with the development of new sorbents and will be used widely for analyses of plant hormones in the future. 
(iii) Immunoaffinity purification. Immunoaffinity chromatography (IAC) purification is based on antibodyantigen ( $\mathrm{Ab}-\mathrm{Ag})$ reorganization, and this specific interaction can provide extremely selective enrichment of the sample and thus greatly enhance the detection limit of the analysis. The method has been applied in the analysis of plant hormones according to its high purification efficiency $[49,50]$. Some non-specifically absorbed compounds in the plant extracts are eluted, while compounds specifically binding with antibodies are retained on the IAC column and then released with suitable solvents. The IAC method is highly selective for trace levels of analytes in the presence of high concentrations of interfering substances. Thus, IAC purification can strongly increase the selectivity and sensitivity of the analysis. However, plant hormones are haptens, which causes problems for specific recognition by antibodies. Therefore plant hormones are usually conjugated to macromolecules to form antigens. Ulvskov and Nicander developed an IAC method in which antibodies are raised against IAA and cytokinins conjugated to bovine serum albumin (BSA) $[51,52]$. The antibody preparation step is the key factor for establishment of the IAC method because the antibody directly affects the specific affinity of the plant hormones. For small molecules, it is still difficult to prepare homogeneous and specific antibodies. Nevertheless, IAC has very broad potential for purification of plant hormones because of its high selectivity and high sensitivity.

\section{Techniques for analyses}

The development of a high-sensitivity and -selectivity detection method is critical to obtain information on the endogenous plant hormone concentration because plant hormones are present in very low physiological concentrations against a background of a wide range of more abundant primary and secondary metabolites. The information is fundamental for understanding the role of plant hormones in signaling transduction networks and their molecular mechanisms. Despite application of multistep purifications to crude plant extracts, there are still a large number of interfering substances in the samples to be analyzed. An increasing number of novel techniques are being developed for determination of plant hormones.

\subsection{Immunoassay}

Immunoassay techniques applied for plant hormone determination include radioimmunoassay (RIA) and enzymelinked immunosorbent assay (ELISA). Both RIA and ELISA use radioactively labeled antigen $\left(\mathrm{Ag}^{*}\right)$ and enzyme-coupled antigen $\left(\mathrm{Ag}^{*}\right)$ to bind with an antibody to form the specific antigen-antibody ( $\mathrm{Ag}^{*}$ - $\left.\mathrm{Ab}\right)$ complex, following which the hormones are quantified by measurement of radioactivity and enzyme activity. Immunoassay is a highly specific detection method because of the specific $\mathrm{Ab}-\mathrm{Ag}$ binding property, and is a sensitive and relatively inexpensive alternative for estimation of plant hormone levels [53-55]. The specificity of the Ab-Ag interaction is the key factor affecting quantification results. Two aspects affect the plant hormone determination. First, plant hormones can stimulate the formation of antibodies when they are conjugated with the carrier because they are haptens, resulting in reduced specificity in an immunoassay system. Second, some interfering substances can be misrecognized by the antibody and cause serious cross reactions. These compounds include plant hormone analogs, intermediates in the plant hormone biosynthesis pathways, and other structurally closely-related compounds. Therefore, it was not until the early 1970s that an immunoassay technique was used for estimation of plant hormone levels. The advantage of the immunoassay method is its lower detection limit and higher sensitivity when applied to determination of plant hormones.

Radioimmunoassay is a very sensitive method to measure concentrations of antigens within the nanomole to picomole range using radiation intensity [56,57]. Special security measures should be taken because radioisotopes such as ${ }^{3} \mathrm{H},{ }^{14} \mathrm{C}$ and ${ }^{125} \mathrm{I}$ are used in RIA. The antisera against bovine serum albumin conjugates of trans-zeatinriboside (tZR) prepared by Weiler have a high affinity to tZR and zeatin [58]. The antisera show negligible cross-reactions with isopentenyladenosine and cis-zeatinriboside, and no cross-reactions with purines and adenosine. However, RIA is now seldom used because of the short stability time of the radiolabeled ligands and potential health hazards.

The immunogens are labeled with enzymes instead of radioisotopes in ELISA. Wang et al. [59] prepared a SA monoclonal antibody using 5-aminosalicylic acid (5-ASA) linked to KLH as an immunogen that was highly specific to SA and even more specific to 5-ASA. Avidin-biotin complex was used in the ELISA assay of zeatin riboside with $100 \mathrm{mg}$ fresh weight of tomato tissue by Maldiney et al. [53]. The antibodies can cross-react with compounds of a similar chemical structure in ELISA, which leads to poor repeatability. Therefore ELISA is not used widely except for brassinosteroids, a very low-concentration steroid plant hormone [60].

Immunosensors, also based on the antigen-antibody interaction, have been applied for determination of plant hormones. An immunosensor is a device comprising an antigen or antibody species coupled to a signal transducer, which detects the binding of the complementary species. The Key Laboratory of Phytohormones of Hunan Agricultural University has made some progress in researching the use of immunosensors of plant hormones [61,62].

Immunoassay is excellent for estimating hormone concentrations in plant tissues because of its advantages of high selectivity, sensitivity and high throughput. Only small amounts of sample tissues and solution volumes are used in immunoassay. Nevertheless, the problem of cross-reactions of the 
antibody remains an obstacle limiting the extensive application of immunoassays for plant hormone quantification.

\subsection{Electrochemical analysis}

The advantages of electrochemical techniques over other analytical techniques are their simplicity, convenience and low cost. Early plant hormone electrochemical studies focused on the electrochemical behavior of abscisic acid, gibberellin, zeatin and kinetin [63-65]. The results showed that plant hormone electrochemical behavior is significantly affected by the $\mathrm{pH}$ and solution matrix, which are more complex in plant samples. Consequently, electrochemical techniques are seldom applied to the determination of practical samples. However, electrochemical biosensors have still attracted interest recently for plant hormone detection on account of their high selectivity, sensitivity and stability. An increasing number of electrochemical biosensors are being developed that can be used in the complicated matrix.

The biorecognition layer, the core component of the electrochemical biosensor, consists of biologically active substances, such as enzymes, microbes, tissues, antibodies or nucleic acids. The chemical signals can be converted to measurable electronic signals on the layer. The current or potential difference is stoichiometrically proportional to the electronically active substance levels. Li et al. [66] developed a novel IAA biosensor using a mung bean sprout leaf-ferrocene modified carbon paste electrode (LFMCE). A certain amount of oxidases of IAA in the leaf of mung bean sprouts can catalyze IAA oxidation. The electrode current of IAA oxidation was used to quantitatively determine IAA content in the plant. A piezoelectric immunosensor, based on the quantitative relationship between the piezoelectric effect and quality change on the surface of the electrode, was also used for the determination of IAA content in a dilute solution [67]. The frequency change of the sensor caused by the antigen binding to the antibody on the electrode surface is linearly related to the logarithm of the concentration of the target compounds.

Biosensor research has made great progress in recent years. The high sensitivity, real-time detection and continuous dynamic monitoring ability of biosensors make them ideal for plant hormone detection. However, poor stability and reproducibility are hurdles to their wide application in practice. Nowadays, biosensors remain at the stage of methodology probes.

\subsection{Chromatography and chromatography/mass spectrometry}

Chromatography basically involves the separation of a mixture dissolved in a mobile phase through a stationary phase. Subtle differences in the partition coefficient of a component can result in differential retention behavior in the stationary phase causing their separation. Gas chromatography and HPLC are common analytical approaches used for the quantitative analysis of plant hormones. Another branch of chromatography, capillary electrophoresis, is also applied in this field, which is based on differences in the charge and frictional forces of analytes under an electric field $[68,69]$. After purification and enrichment, the samples are still difficult for plant hormone determination owing to their complicated matrix, but the high separation efficiency of chromatography and the capability for combination with different detectors makes it an ideal technique for separating plant hormones from the matrix and to provide improved quantitative results. Gas chromatography and HPLC have been used for quantitative analysis of plant hormones since the early 1970s. Chromatography played an important role in the early days. Plant hormones need to be derived to increase their volatility and improve their thermal stability when using GC. The derivatization involves many steps during the sample pretreatment, so this time-consuming aspect should be optimized carefully. HLPC, which can work with polar compounds directly, is more suitable for most plant hormones without derivatization when using a UV detector. The main drawback of using a UV detector is its lower sensitivity [70,71]. Compared with a UV detector, the sensitivity of a fluorescence detector (FLD) is about 2-3 orders of magnitude higher, so FLD is more suitable for plant hormone detection $[21,22,72,73]$. Because the qualification of analytes is only based on the retention time and UV or fluorescence spectroscopy with GC or HPLC combined with these detectors, it is a little dangerous to draw the correct conclusion, because many components may be present in one peak with a complicated matrix. So UV and FLD cannot meet the accurate measurement requirements of trace plant hormone analysis. Gas/liquid chromatographyMS overcomes the disadvantages of traditional chromatography technology and has become a popular tool for quantitative analysis of plant hormones.

Mass spectrometry is an analytical technique that measures the mass-to-charge ratio $(\mathrm{m} / \mathrm{z})$ of charged particles. The analytes are ionized in the ion source, and then formed into an ion beam to pass through the ion guide system, followed by analysis with a mass analyzer based on the $\mathrm{m} / \mathrm{z}$ ratio. Molecular fragments are also obtained via different dissociation techniques such as collision induced dissociation. The $m / z$ separation capability of a mass analyzer enhances the analysis of a plant extract after purification and enrichment by liquid partition or SPE. The researcher can monitor characteristic ions produced by MS with extremely high selectivity. Single quadrupole GC/MS and LC/MS applied in plant hormone analysis is based on the selective ion monitoring (SIM) mode, in which only specific characteristic ions of the target compound are measured. SIM provides higher sensitivity and selectivity than monitoring all ions simultaneously, a data acquisition mode named full scan. Chemical ionization and electron ionization (EI) are two widely used ionization methods for GC-MS analysis $[13,16,74,75]$. However, EI typically uses electrons at 70 
$\mathrm{eV}$, which yields more fragments ions. Identification of the target compound is difficult in a complicated matrix [76]. The development of atmospheric pressure ionization technology has significantly expanded the application of LC/MS. Electrospray ionization (ESI) and atmospheric pressure chemical ionization (APCI) overcome the shortcomings of EI and have become two widely used ionization interfaces in LC/MS. Currently, ESI is the most common ion source used in LC/MS because of its higher sensitivity and lower background [77], although frit-fast atom bombardment has also been used for analysis of cytokinins [78]. For some low-concentration plant hormones, the complicated matrix still interferes with the analysis, even using a SIM scan mode. A better alternative is the multiple reactions monitoring (MRM) mode with higher selectivity based on the tandem mass spectrometry (MS/MS) technique, which can be performed in either time or space, corresponding to triple quadrupole mass spectrometry (QQQ MS) and ion trap mass spectrometry (IT MS), respectively. In MRM a precursor mass ion is selected in the first-stage quadrupole and then fragmented in the collision cell to yield diagnostic product ions filtered by the third-stage quadrupole. A signal is detected only when the selected precursor ion passes the first-stage quadrupole and the selected product ion passes the third-stage quadrupole; thus each ionized compound gives a distinct precursor-to-product ion transition in the MRM mode that is diagnostic for the presence of a particular compound in an extract. Figure 1 gives the principle of isotope dilution using MRM mode on triple quadrupole mass spectrometer. The MRM mode shows higher selectivity and sensitivity than SIM and can detect the target plant hormones quickly [79,80]. Highly sensitive MS techniques have greatly reduced the amount of sample tissues required. Increasingly, protocols developed are based on LC-MS/MS [8,12,37,80]. Müller et al. [81] developed a GC-MS/MS method for rapid and accurate quantification of SA, JA, IAA and ABA simultaneously from 20 mg plant tissues. A nano-electrospray (nanoESI) source, which can generate product ions with higher efficiency using nanoliter flow rates $(1 \mathrm{~nL} / \mathrm{min}$ to $1 \mu \mathrm{L} / \mathrm{min})$ and nanocolumns [82], is also employed in quantitative analysis of plant hormones. The sensitivity of nanoESI-MS has been significantly improved. Some problems associated with a nano-ESI source are the fragile spray nozzle and poor sta- bility and reproducibility compared with a conventional ESI source. Moreover, the unique matching chromatography combined with MS still needs to be improved. These shortcomings hinder the wide application of nanoESI [83].

A milestone in LC system development is the application of fine particulate in separation science in recent years, which overcomes the limitation of conventional HPLC. This new type of LC is named ultra-performance liquid chromatography (UPLC). The column is packed with $1.7 \mu \mathrm{m}$ particulate and can tolerant 1000 bar pressure. The use of smaller particulate empowered UPLC with higher separation efficiency in a very short program. The total run time of the UPLC method may take several minutes only, whereas that of the HPLC method may take at least dozens of minutes. The UPLC technique was introduced to plant hormone analyses recently. Novak et al. [10] separated 32 cytokinin derivatives in less than $10 \mathrm{~min}$, which was almost 4-fold faster than HPLC. More sample information will be obtained when UPLC is combined with time-of-flight mass spectrometry (TOF-MS) because of its high separation efficiency; indeed, an UPLC-TOF-MS system for the analysis of plant hormones has been reported recently [84].

There are two means of improving plant hormone quantitative analysis results. First, isotope dilution should be used because multistep pretreatment is involved. Stable isotope-labeled plant hormones, usually labeled with ${ }^{2} \mathrm{H},{ }^{13} \mathrm{C}$ and ${ }^{15} \mathrm{~N}$, are added to the ground plant tissues before extraction. These isotope-labeled internal standards have almost identical physicochemical properties and chemical stability with endogenous plant hormones, so they can correct the plant hormone loss error during sample pretreatment and have almost the same chromatography and MS behavior [85]. The contents of plant hormones are determined by comparison of the MS response between the internal standards and the endogenous plant hormones under a SIM or MRM mode. The corresponding stable isotope-labeled plant hormones, such as ${ }^{2} \mathrm{H}_{2}$-IAA, ${ }^{2} \mathrm{H}_{6}$-ABA, ${ }^{2} \mathrm{H}_{5}$-JA and ${ }^{2} \mathrm{H}_{4}$-SA, are used widely $[8,12,37,80]$. Second, derivatization is needed to enhance sensitivity in some cases. High sensitivity is a prerequisite for plant hormone analysis. Some lowconcentration plant hormones are difficult to quantify even when separated and purified by multistep pretreatments. Thus, derivatization can improve sensitivity dramatically. Chemical derivatization in GC/MS is usually aimed at in-
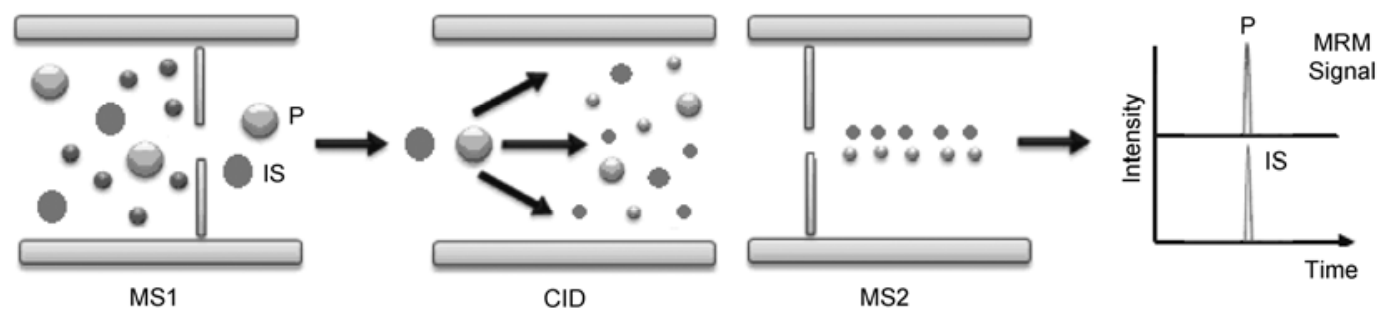

Figure 1 The principle of isotope dilution using a multiple reactions monitoring (MRM) mode on a triple quadrupole mass spectrometer. MS1, monitoring precursor ion; CID, collision cell; MS2, monitoring product ion; IS, stable isotope-labeled internal standard; P, plant hormone. 
Table 3 Derivatization methods for plant hormones

\begin{tabular}{|c|c|c|c|}
\hline Plant hormones & Reagent $t^{a}$ & Derivatization protocols & References \\
\hline \multirow{5}{*}{ IAA } & Diazomethane & Sample was methylated using a stream of diazomethane gas & [30] \\
\hline & & $\begin{array}{l}\text { Sample was dissolved in } 10 \%(\mathrm{v} / \mathrm{v}) \text { methanol in diethylether and was } \\
\text { methylated by reaction with diazomethane for } 10 \mathrm{~min} \text { at } 0^{\circ} \mathrm{C}\end{array}$ & [7] \\
\hline & & Incubate at room temperature in methanol solution & {$[81,90]$} \\
\hline & MTBSTFA & Incubate for $60 \mathrm{~min}$ at $100^{\circ} \mathrm{C}$ & [94] \\
\hline & BETAB & $\begin{array}{c}\text { Samples were dissolved in } 20 \mu \mathrm{L} \text { aliquot of water, } 4 \mu \mathrm{L} 500 \mathrm{mmol} / \mathrm{L} \\
\text { bromocholine in } 70 \% \text { acetonitrile and } 0.8 \mu \mathrm{L} \text { triethylamine, and then } \\
\text { heated at } 80^{\circ} \mathrm{C} \text { for } 130 \mathrm{~min}\end{array}$ & [87] \\
\hline \multirow{4}{*}{ ABA } & Diazomethane & $\begin{array}{l}\text { Sample was dissolved in } 10 \%(\mathrm{v} / \mathrm{v}) \text { methanol in diethylether and was } \\
\text { methylated by reaction with diazomethane for } 10 \mathrm{~min} \text { at } 0^{\circ} \mathrm{C}\end{array}$ & [7] \\
\hline & & Incubate at room temperature in methanol solution & [81] \\
\hline & MTBSTFA & Incubate for $60 \mathrm{~min}$ at $100^{\circ} \mathrm{C}$ & [94] \\
\hline & BETAB & $\begin{array}{l}\text { Samples was dissolved in } 20 \mu \mathrm{L} \text { aliquot of water, } 4 \mu \mathrm{L} 500 \mathrm{mmol} / \mathrm{L} \text { bro- } \\
\text { mocholine in } 70 \% \text { acetonitrile and } 0.8 \mu \mathrm{L} \text { triethylamine, and then heated } \\
\text { at } 80^{\circ} \mathrm{C} \text { for } 130 \mathrm{~min}\end{array}$ & [87] \\
\hline \multirow{2}{*}{ JA } & MTBSTFA & Incubate for $60 \mathrm{~min}$ at $100^{\circ} \mathrm{C}$ & [94] \\
\hline & Diazomethane & Incubate at room temperature in methanol solution & [81] \\
\hline \multirow{3}{*}{ SA } & MTBSTFA & Incubate for $60 \mathrm{~min}$ at $100^{\circ} \mathrm{C}$ & [94] \\
\hline & Diazomethane & Incubate at room temperature in methanol solution & [81] \\
\hline & MTBSTFA & Incubate for $60 \mathrm{~min}$ at $100^{\circ} \mathrm{C}$ & [94] \\
\hline \multirow{5}{*}{ CTKs } & $\begin{array}{c}\text { ACN: acetic anhydride: NMIM (100:10:1) } \\
\text { MSHFBA }\end{array}$ & Incubate for $30 \mathrm{~min}$ at room temperature & [75] \\
\hline & BTMSA & Incubate for $30 \mathrm{~min}$ at $90^{\circ} \mathrm{C}$ & [94] \\
\hline & Propionic anhydride & Incubate for $5 \mathrm{~min}$ at $60^{\circ} \mathrm{C}$ in acetonitrile solution & [91] \\
\hline & BETAB & $\begin{array}{l}\text { Sample was derivatized by heating to } 37^{\circ} \mathrm{C} \text { for } 30 \mathrm{~min} \text { in a mixture of } \\
\text { acetonitrile, } N \text {-methylimidazole and propionic anhydride }(10: 6: 3)\end{array}$ & [88] \\
\hline & & $\begin{array}{c}\text { Samples were dissolved in } 20 \mu \mathrm{L} \text { aliquot of water, } 4 \mu \mathrm{L} 500 \mathrm{mmol} / \mathrm{L} \\
\text { bromocholine in } 70 \% \text { acetonitrile and } 0.8 \mu \mathrm{L} \text { triethylamine, and then } \\
\text { heated at } 80^{\circ} \mathrm{C} \text { for } 130 \mathrm{~min}\end{array}$ & [87] \\
\hline \multirow[t]{2}{*}{$\mathrm{GA}_{1}, \mathrm{~A}_{3}$} & BSTFA & $\begin{array}{l}\text { GA methyl esters were silylated with a solution of BSTFA and pyridine at } \\
\qquad 80^{\circ} \mathrm{C} \text { for } 15 \mathrm{~min}\end{array}$ & [18] \\
\hline & & Incubate for $30 \mathrm{~min}$ at $90^{\circ} \mathrm{C}$ & [94] \\
\hline GAs & BETAB & $\begin{array}{c}\text { The samples was dissolved in } 20 \mu \mathrm{L} \text { aliquot of water, } 4 \mu \mathrm{L} 500 \mathrm{mmol} / \mathrm{L} \\
\text { bromocholine in } 70 \% \text { acetonitrile and } 0.8 \mu \mathrm{L} \text { triethylamine, and then } \\
\text { heated at } 80^{\circ} \mathrm{C} \text { for } 130 \mathrm{~min}\end{array}$ & [87] \\
\hline BRs & Dansyl-3-aminophenylboronic acid & $\begin{array}{c}\text { Sample was derivatized by heating to } 62^{\circ} \mathrm{C} \text { for } 30 \mathrm{~min} \text { in a mixture of } \\
\text { pyridine and acetonitrile }(1: 19)\end{array}$ & [86] \\
\hline
\end{tabular}

a) MTBSTFA, $N$-methyl- $N$-(tert-butyldimethylsilyl)trifluoroacetamide; MSHFBA, $N$-methyl- $N$-(trimethylsilyl)heptafluorobutyramide; BSTFA, $N$,O-bis (trimethylsilyl)trifluoroacetamide; BTMSA, bis(trimethylsilyl)acetylene; BETAB, (2-bromoethy1)trimethyl-ammonium bromide.

creasing the volatility of plant hormones, so that the derived plant hormones are more suitable for GC/MS analysis. Although LC/MS can analyze plant hormones directly, derivatization is employed to improve ionization efficiency of the derived hormones, which can improve sensitivity by 10 to 1000-fold compared with underived hormones, and so has received increasing attention for the analysis of plant hormones [86-88].

Among the huge family of derivatization reagents, diazomethane is one of the commonly used reagents with a high reaction speed and fewer by-products. However, diazomethane should be used with extreme caution because of its toxicity and explosive nature [89]. The detection limit for IAA can increase 3-fold after the samples are methylated with diazomethane [90]. Other derivatization reagents, such as trimethylsilyl, t-butyldimethylsilyl, trifluoroacetyl and acetyl, are also employed in plant hormone analysis [75,91-93]. The polar plant hormone, cytokinins, can be acetylated and analyzed by GC/MS. Björkman and Tillberg [75] developed such a GC/MS derivatization method exhibiting high recovery, a clean background, and fewer byproducts. However, the acetylated O-glucosides were unstable in GC/MS, and the derivatives of zeatin and dihydrozeatin showed only weak molecular ions, so determination of novel cytokinins with this method is difficult. Brassinosteroids are difficult to quantify using either GC/MS or LC/MS. The C22-C23 diol moiety can be selectively derived using dansyl-3-aminophenylboronic acid to form dansyl-3-amin- ophenylboronates with a limit of detection of 125 attomole, thus Svatoš et al. [86] provide hope that 
further quantification of brassinosteroids is possible with LC/MS. Thus, for GC/MS analysis, derivatization not only enhances volatility but also improves stability of target compounds; precolumn derivatizations for LC/MS hormone analyses are used to produce stronger ion currents and increase the sensitivity. Different derivatization methods for plant hormones are summarized in Table 3. Appropriate choice of derivatization reagents depends on both the plant hormone and the corresponding derivatives' physiochemical properties, such as stability, boiling point and molecular polarity. To develop an optimum program of derivatization, it is necessary to investigate the parameters including derivative reagents, reaction time and temperature systematically.

\section{Summary and perspective}

Analysis of plant hormones is difficult because of their low concentration in plant extracts, which also contain a wide variety of interference substances. To improve identification and quantification of endogenous plant hormones, a suitable method should be employed to extract and enrich these trace compounds from plant tissues. These pretreatment steps can minimize the matrix effect of interfering substances. Sensitivity is still the key consideration in this field. Each analytical method has its own advantages and disadvantages. All methods based on the antigen-antibody specific interaction have extremely high theoretical sensitivity and specificity for purification and detection of hormones, but cross-reactions cause many problems. Electrochemical analysis, the most suitable real-time quantification approach, is limited by its instability, poor reproducibility and short service life. Only chromatography combined with mass spectrometry, especially triple quadrupole mass spectrometry, has rapidly become a highly sensitive, selective tool for plant hormone analyses in practice. The capability of combination with $\mathrm{CE}, \mathrm{GC}$ or LC broadens detection with mass spectrometry. In particular, GC or LC coupled with tandem mass spectrometry not only significantly improves the sensitivity but also provides structural information based on fragmentation patterns. LC-MS/MS has become the most popular tool in plant hormone analysis because hormones can be analyzed directly. However, the high cost of instruments and the consumption of stable isotope-labeled internal standards also hinder its wider application. Nowadays, an increasing number of scientists realize that precise and accurate quantitative plant hormone information in model plants will help them understand the molecular mechanism by which hormones regulate plant growth, development and responses to environmental stresses. Plant hormones are the cornerstone of molecular breeding and the key to open the door of modern agriculture. However, plant hormone analysis remains a bottleneck for plant biologists, especially for some low-concentration plant hormones. The trends for the analysis of plant hormones should include the following points. First, quantify multiple classes of plant hormones simultaneously using a simple and sensitive method. Increasing evidence has shown that many biochemical events are not determined by a single plant hormone [95]. Scientists have adjusted their focus from single plant hormone function to plant hormone crosstalk [96]. Second, development of sensitive, in situ, transient, dynamic analytical methods for determining trace levels of different plant hormones in a live plant. This is essential for elucidating the role and function of specific metabolites in diverse biological systems. Third, label plant hormones with stable isotopes and synthesize plant hormones for precise quantitative analysis. Many chiral atoms present in plant hormone molecular structures are challenge for organic chemists. Finally, develop of high-throughput separation and enrichment methods for plant hormone purification and quantification. These methods can handle multiple samples in parallel. It is predictable that the development of new instruments will play an important role in the quantitative analysis and structural identification of plant hormones. As an increasing number of novel plant hormones are discovered, our knowledge of plant hormones will be expanded.

We thank Dr. LUO HaiYing for checking the manuscript. This work was supported by the National Natural Science Foundation of China (90917017 and 90817008).

1 Davies P J. Plant Hormones: Physiology, Biochemistry and Molecular Biology. Dordrecht, Netherlands: Kluwer, 1995. 1-12

2 Harman G E, Howell C R, Viterbo A, et al. Trichoderma species-opportunistic avirulent plant symbionts. Nat Rev Microbiol, 2004, 2: $43-56$

3 Bray E A. Abscisic acid regulation of gene expression during water-deficit stress in the era of the Arabidopsis genome. Plant Cell Environ, 2002, 25: 153-161

4 Durrant W E, Dong X. Systemic acquired resistance. Annu Rev Phytopathol, 2004, 42: 185-209

5 Hao J J, Kang C L. Plant Biology. Beijing: Chemical Industry Press, 2005. 160

6 Barkawi L S, Tam Y Y, Tillman J A, et al. A high-throughput method for the quantitative analysis of indole-3-acetic acid and other auxins from plant tissue. Anal Biochem, 2008, 372: 177-188

7 Vine J H, Noiton D, Plummer J A, et al. Simultaneous quantitation of indole 3-acetic acid and abscisic acid in small samples of plant tissue by gas chromatography-mass spectrometry/selected ion monitoring. Plant Physiol, 1987, 85: 419-422

8 Ross A R S, Ambrose S J, Cutler A J, et al. Determination of endogenous and supplied deuterated abscisic acid in plant tissues by high-performance liquid chromatography-electrospray ionization tandem mass spectrometry with multiple reaction monitoring. Anal Biochem, 2004, 329: 324-333

9 Vilaró F, Canela-Xandri A, Canela R. Quantification of abscisic acid in grapevine leaf (Vitis vinifera) by isotope-dilution liquid chromatography-mass spectrometry. Anal Bioanal Chem, 2006, 386: 306-312

10 Novak O, Hauserova E, Amakorova P, et al. Cytokinin profiling in plant tissues using ultra-performance liquid chromatography-electrospray tandem mass spectrometry. Phytochemistry, 2008, 69: 2214-2224

11 Gawronska H, Yang Y Y, Furukawa K, et al. Effects of low irradiance stress on gibberellin levels in pea-seedlings. Plant Cell Physiol, 1995, 36: 1361-1367

12 Kristl J, Veber M, Krajničič B, et al. Determination of jasmonic acid 
in Lemna minor (L.) by liquid chromatography with fluorescence detection. Anal Bioanal Chem, 2005, 383: 886-893

13 Engelberth J, Schmelz E A, Alborn H T, et al. Simultaneous quantification of jasmonic acid and salicylic acid in plants by vapor-phase extraction and gas chromatography-chemical ionization-mass spectrometry. Anal Biochem, 2003, 312: 242-250

14 Cao J, Murch S J, O'Brien R, et al. Rapid method for accurate analysis of melatonin, serotonin and auxin in plant samples using liquid chromatography-tandem mass spectrometry. J Chromatogr A, 2006, 1134: 333-337

15 Liu B F, Zhong X H, Lu Y T. Analysis of plant hormones in tobacco flowers by micellar electrokinetic capillary chromatography coupled with on-line large volume sample stacking. J Chromatogr A, 2002, 945: 257-265

16 Zhang F J, Jin Y J, Xu X Y, et al. Study on the extraction, purification and quantification of jasmonic acid, abscisic acid and indole-3acetic acid in plants. Phytochem Anal, 2008, 19: 560-567

17 Moritz T, Olsent J E. Comparison between high resolution selected ion monitoring, selected reaction monitoring and four-sector tandem mass spectrometry quantitative analysis of gibberellins in milligram amounts of plant tissue. Anal Chem, 1995, 67: 1711-1716

18 Symons G M, Reid J B. Hormone levels and response during de-etiolation in Pea. Planta, 2003, 216: 422-431

19 Jager C E, Symons G M, Nomura T, et al. Characterization of two brassinosteroid C-6 oxidase genes in Pea. Plant Physiol, 2007, 143: 1894-1904

20 Zullo M A T, Adam G. Brassinosteroid phytohormones-structure, bioactivity and applications. Braz J Plant Physiol, 2002, 14: 143-181

21 Rozhon W, Petutschnig E, Wrzaczek M, et al. Quantification of free and total salicylic acid in plants by solid-phase extraction and isocratic high-performance anion-exchange chromatography. Anal Bioanal Chem, 2005, 382: 1620-1627

22 Eshita S M. 3-hydroxybenzoic acid as an internal standard for the high-pressure liquid chromatography quantitation of salicylic acid in plants. Anal Biochem, 2001, 289: 99-102

23 Enyedi A J, Yalpani N, Silverman P, et al. Localization, conjugation and function of salicylic acid in tobacco during the hypersensitive reaction to tobacco mosaic virus. Proc Natl Acad Sci USA, 1992, 89: 2480-2484

24 Bieleski R L. The problem of halting enzyme action when extracting plant tissues. Anal Biochem, 1964, 9: 431-442

25 Horgan R, Scott I M. Cytokinins. In: Rivier L, Crozier A, eds. The Principles and Practice of Plant Hormone Analysis. London: Academic Press, 1987. 304-365

26 Laloue M, Terrine C, Gawer M. Cytokinins: Formation of the nucleoside-50-triphosphate in tobacco and Acer cells. FEBS Lett, 1974, 46: 45-50

27 Hoyerová K, Gaudinová A, Malbeck J, et al. Efficiency of different methods of extraction and purification of cytokinins. Phytochemistry, 2006, 67: 1151-1159

28 Izumi Y, Okazawa A, Bamba T, et al. Development of a method for comprehensive and quantitative analysis of plant hormones by highly sensitive nanoflow liquid chromatography-electrospray ionization-ion trap mass spectrometry. Anal Chim Acta, 2009, 648: 215-225

$29 \mathrm{Wu}$ Y, Hu B. Simultaneous determination of several phytohormones in natural coconut juice by hollow fiber-based liquid-liquid-liquid microextraction-high performance liquid chromatography. J Chromatogr A, 2009, 1216: 7657-7663

30 Ribnicky D M, Cooke T J, Cohen J D. A microtechnique for the analysis of free and conjugated indole-3-acetic acid in milligram amounts of plant tissue using a benchtop gas chromatograph-mass spectrometer. Planta, 1998, 204: 1-7

31 Wijayanti L, Kobayashi M, Fujioka S, et al. Identification and quantification of abscisic acid, indole-3-acetic acid and gibberllins in phloem exudates of Pharbitis nil. Biosci Biotech Biochem, 1995, 59: 1533-1535

32 Dunlap J R, Guinn G. A simple purification of indole-3-acetic acid and abscisic acid for GC-SIM-MS analysis by microfiltration of aqueous samples through nylon. Plant Physiol, 1989, 90: 197-201

33 Varbanova M, Yamaguchi S, Yang Y, et al. Methylation of gibberellins by Arabidopsis GAMT1 and GAMT2. Plant Cell, 2007, 19: 32-45

34 Astot C, Dolezal K, Moritz T, et al. Deuterium in vivo labelling of cytokinins in Arabidopsis thaliana analysed by capillary liquid chromatography/frit-fast atom bombardment mass spectrometry. J Mass Spectrom, 2000, 35: 13-22

35 Novák O, Tarkowski P, Tarkowská D, et al. Quantitative analysis of cytokinins in plants by liquid chromatography-single-quadrupole mass spectrometry. Anal Chim Acta, 2003, 480: 207-218

36 Ma Z, Ge L, Lee A S Y, et al. Simultaneous analysis of different classes of phytohormones in coconut (Cocos nucifera L.) water using high-performance liquid chromatography and liquid chromatography-tandem mass spectrometry after solid-phase extraction. Anal Chim Acta, 2008, 610: 274-281

37 Matsuda F, Miyazawa H, Wakasa K, et al. Quantification of indole-3-acetic acid and amino acid conjugates in rice by liquid chromatography-electrospray ionization-tandem mass spectrometry. Biosci Biotechnol Biochem, 2005, 69: 778-783

38 Rhijn J A V, Heskamp H H, Davelaar E, et al. Quantitative determination of glycosylated and aglycon isoprenoid cytokinins at sub-picomolar levels by microcolumn liquid chromatography combined with electrospray tandem mass spectrometry. J Chromatogr A, 2001, 929: 31-42

39 Ge L, Yong J W H, Goh N K, et al. Identification of kinetin and kinetin riboside in coconut (Cocos nucifera $\mathrm{L}$.) water using a combined approach of liquid chromatography-tandem mass spectrometry, high performance liquid chromatography and capillary electrophoresis. J Chromatogr B, 2005, 829: 26-34

40 Ge L, Yong J W H, Tan S N, et al. Analysis of some cytokinins in coconut (Cocos nucifera L.) water by micellar electrokinetic capillary chromatography after solid-phase extraction. J Chromatogr A, 2004, 1048: 119-126

41 Ge L, Yong J W H, Tan S N, et al. Analysis of cytokinin nucleotides in coconut (Cocos nucifera L.) water using capillary zone electrophoresis-tandem mass spectrometry after solid-phase extraction. J Chromatogr A, 2006, 1133: 322-331

42 Hou S, Zhu J, Ding M, et al. Simultaneous determination of gibberellic acid, indole-3-acetic acid and abscisic acid in wheat extracts by solid-phase extraction and liquid chromatography-electrospray tandem mass spectrometry. Talanta, 2008, 76: 798-802

43 Dobrev P I, Havlíček L, Vágner M, et al. Purification and determination of plant hormones auxin and abscisic acid using solid phase extraction and two-dimensional high performance liquid chromatography. J Chromatogr A, 2005, 1075: 159-166

44 Smith J L, Moraes C M D, Mescher M C. Jasmonate-and salicylate-mediated plant defense responses to insect herbivores, pathogens and parasitic plants. Pest Manag Sci, 2009, 65: 497-503

45 Baldwin I T, Zhang Z P, Diab N, et al. Quantification, correlations and manipulations of wound-induced changes in jasmonic acid and nicotine in Nicotiana sylvestris. Planta, 1997, 201: 397-404

46 Chen H, Wilkerson C G, Kuchar J A, et al. Jasmonate-inducible plant enzymes degrade essential amino acids in the herbivore midgut. Proc Natl Acad Sci USA, 2005, 102: 19237-19242

47 Koo A J K, Gao X, Daniel J A, et al. A rapid wound signal activates the systemic synthesis of bioactive jasmonates in Arabidopsis. Plant J, 2009, 59: 974-986

48 Liu H T, Li Y F, Luan T G, et al. Simultaneous determination of phytohormones in plant extracts using SPME and HPLC. Chromatographia, 2007, 66: 515-520

49 Vankova R, Gaudinova A, Sussenbekova H, et al. Comparison of oriented and random antibody immobilization in immunoaffinity chromatography of cytokinins. J Chromatogr A, 1998, 811: 77-84

50 Hauserová E, Swaczynová J, Doležal K, et al. Batch immunoextraction method for efficient purification of aromatic cytokinins. J Chromatogr A, 2005, 1100: 116-125

51 Ulvskov P, Marcussen J, Rajagopal R, et al. Immunoaffinity purification of indole-3-acetamide using monoclonal antibodies. Plant Cell Physiol, 1987, 28: 937-945

52 Nicander B, Stahl U, Bjorkman P O, et al. Immunoaffinity co-purification of cytokinins and analysis by high-performance liquid chromatography with ultraviolet-spectrum detection. Planta, 1993, 189: 312-320

53 Maldiney R, Leroux B, Sabbagh I, et al. A biotin-avidin-based enzyme immunoassay to quantify three phytohormones: Auxin, abscisic 
acid and zeatin-riboside. J Immunol Methods, 1986, 90: 151-158

54 Morris R O, Blevins D G, Dietrich J T, et al. Cytokinins in plant pathogenic bacteria and developing cereal grains. Aust J Plant Physiol, 1993, 20: 621-637

55 Yong J W H, Wong S C, Letham D S, et al. Effects of elevated [CO2] and nitrogen nutrition on cytokinins in the xylem sap and leaves of cotton. Plant Physiol, 2000, 124: 767-779

56 Grayling A, Hanke D E. Cytokinins in exudates from leaves and roots of red Perilla. Phytochemistry, 1992, 31: 1863-1868

57 Cook N C, Bellstedt D U, Jacobs G. Endogenous cytokinins distribution patterns at budburst in Granny Smith and Braeburn apple shoots in relation to bud growth. Sci Hort, 2001, 87: 53-63

58 Weiler E W. Radioimmunoassays for trans-zeatin and related cytokinins. Planta, 1980, 149: 155-162

59 Wang S C, Li G J, Kai X, et al. Preparation and application of monoclonal antibodies specific for salicylic acid. Acta Bot Sin, 2001, 43: 1207-1210

60 Swaczynová J, Novák O, Hauserová E, et al. New techniques for the estimation of naturally occurring brassinosteroids. J Plant Growth Regul, 2007, 26: 1-14

$61 \mathrm{Li} \mathrm{J}, \mathrm{Wu} \mathrm{Z}$ Y, Xiao L T, et al. A novel piezoelectric biosensor for the detection of phytohormones-indole acetic acid. Analyt Sci, 2002, 18: 1-5

62 Li C X, Li J, Xiao L T, et al. Amperometric immunosensor based on mercapto carboxylic acid self assembled monolayer for phytohormone indoleacetic acid assay. Acta Chim Sin, 2003, 61: 790-794

63 Wang H, Hu S S, Zhou X Y J. Voltammetric behavior of plant hormone abscisic acid. Wuhan Univ, 1997, 43: 721-724

64 Liu X H, Wang L S, Jiang Z L. A study on the 2.5 order differential voltammetric method for gibberellin and its application. J Anal Sci, 1997, 13: 222-224

65 Jiang Z W, Jiang T B, Ju C Q, et al. Voltammetric behavior of zeatin and kinetin. Chem J Chin Univ, 1994, 15: 355-359

66 Li C X, Li J, Xiao L T, et al. Study on the electrochemical biosensor for photohormone indole 3-acetic acid. J Anal Sci, 2003, 19: 205-208

67 Li J, Wu Z Y, Xiao L T. A novel piezoelectric biosensor for the detection of phytohormone $\beta$-indole acetic acid. Anal Sci, 2002, 18: 403-407

68 Ge L, Peh C Y C, Yong J W H, et al. Analyses of gibberellins by capillary electrophoresis-mass spectrometry combined with solidphase extraction. J Chromatogr A, 2007, 1159: 242-249

69 Ge L, Yong J W H, Tan S N, et al. Determination of cytokinins in coconut (Cocos nucifera $\mathrm{L}$.) water using capillary zone electrophoresis-tandem mass spectrometry. Electrophoresis, 2006, 27: 2171-2181

70 Albrecht T, Kehlen A, Stahl K, et al. Immunoaffinity co-purification of cytokinins and analysis by high-performance liquid chromatography liquid chromatography with ultraviolet-spectrum detection. Planta, 1993, 191: 86-94

71 Gamoh K, Kitsuwa T, Takatsuto S, et al. Determination of trace brassinosteroids by high performance liquid chromatography. Anal Sci, 1988, 4: 533-535

72 Verberne M C, Brouwer N, Delbianco F, et al. Method for the extraction of the volatile compound salicylic acid from tobacco leaf material. Phytochem Anal, 2002, 13: 45-50

73 Fu J H, Chu J F, Wang J D, et al. Determination of auxin in Arabidopsis thaliana by solid phase extraction and high performance liquid chromatography with fluorescence detection. Chin J Anal Chem, 2009, 37: 1324-1327

74 Schmelz E A, Engelberth J, Alborn H T, et al. Airborne signals prime plants against insect herbivore attack. Proc Natl Acad Sci USA, 2003, 100: 10552-10557

75 Björkman P O, Tillberg E. Acetylation of cytokinins and modified adenine compounds: A simple and non-destructive derivatization method for gas chromatography-mass spectrometric analysis phytochemical analysis. Phytochem Anal, 1996, 7: 57-68

76 Ikekawa N, Takatsuto S. Microanalysis of brassinosteroids in plants by gas chromatography/Mass spectrometry. Mass Spectroscopy, 1984,
32: 55-70

77 Tarkowski P, Ge L, Yong J W H, et al. Analytical methods for cytokinins. Trends Anal Chem, 2009, 28: 323-335

78 Astot C, Dolezal K, Moritz T, et al. Precolumn derivatisation and capillary liquid chromatographic/frit-fast atom bombardment mass spectrometry analysis of cytokinins in Arabidopsis thaliana. J Mass Spectrom, 1998, 33: 892-902

79 Pan X Q, Welti R, Wang X M. Simultaneous quantification of major phytohormones and related compounds in crude plant extracts by liquid chromatography-electrospray tandem mass spectrometry. Phytochemistry, 2008, 69: 1773-1781

80 Segarra G, Jáuregui O, Casanova E, et al. Simultaneous quantitative LC-ESI-MS/MS analyses of salicylic acid and jasmonic acid in crude extracts of Cucumis sativus under biotic stress. Phytochemistry, 2006, 67: 395-401

81 Müller A, Düchting P, Weiler E W. A multiplex GC-MS /MS technique for the sensitive and quantitative single-run analysis of acidic phytohormones and related compounds and its application to Arabidopsis thaliana. Planta, 2002, 216: 44-56

82 Izumi Y, Okazawa A, Bamba T, et al. Development of a method for comprehensive and quantitative analysis of planthormones by highly sensitive nanoflow liquid chromatography-electrospray ionization-ion trap mass spectrometry. Anal Chim Acta, 2009, 648: 215-225

83 Pramanik B N, Ganguly A K, Gross M L. Applied Electrospray Mass Spectrometry. Beijing: Chemical Industry Press, 2005. 132

84 Glauser G, Grata E, Dubugnon L, et al. Spatial and temporal dynamics of jasmonate synthesis and accumulation in Arabidopsis in response to wounding. J Biol Chem, 2008, 283: 16400-16407

85 Rivier L, Crozier A. Principles and Practice of Plant Hormones Analysis, 1 and 2. London: Academic Press, 1987

86 Svatoš A, Antonchick A, Schneider B. Determination of brassinosteroids in the sub-femtomolar range using dansyl-3-aminophenylboronate derivatization and electrospray mass spectrometry. Rapid Commun Mass Spectrom, 2004, 18: 816-821

87 Kojima M, Kamada-Nobusada T, Komatsu H. Highly sensitive and high-throughput analysis of plant hormones using MS-Probe modification and liquid chromatography-tandem mass spectrometry: An application for hormone profiling in Oryza sativa. Plant Cell Physiol, 2009, 50: 1201-1214

88 ordström A, Tarkowski P, Tarkowska D, et al. Derivatization for LC-electrospray ionization-MS: A tool for improving reversed-phase separation and ESI responses of bases, ribosides, and intact nucleotides. Anal Chem, 2004, 76: 2869-2877

89 Wang L, Wang Z F. Chromatographic Analysis and Sample Handling. Beijing: Chemical Industry Press, 2006. 94

90 Prinsen E, Dongen W V, Esmans E L, et al. HPLC linked electrospray tandem mass spectrometry: A rapid and reliable method to analyse indole-3-acetic acid metabolism in bacteria. J Mass Spectrom, 1997, 32: 12-22

91 Most B H, Williams J C, Parker K J. Gas chromatography of cytokinins. J Chromatogr A, 1968, 38: 136-138

92 Hocart C H, Wong O C, Letham D S, et al. Mass spectrometry and chromatography of t-butyldimethylsilyl derivatives of cytokinin bases. Anal Biochem, 1986, 153: 85-96

93 Ludewig M, Dörffling K, König W A. Electron-capture capillary gas chromatography and mass spectrometry of trifluoroacetylated cytokinins. J Chromatogr A, 1982, 243: 93-98

94 Birkemeyer C, Kolasa A, Kopka J. Comprehensive chemical derivatization for gas chromatography-mass spectrometry-based multitargeted profiling of the major phytohormones. J Chromatogr A, 2003, 993: 89-102

95 Santner A, Estelle M. Recent advances and emerging trends in plant hormone signaling. Nature, 2009, 456: 1071-1078

96 Rost T L, Weier T E. Botany, an Introduction to Plant Biology. New York: Wiley, 1979: 155-170

Open Access This article is distributed under the terms of the Creative Commons Attribution License which permits any use, distribution, and reproduction in any medium, provided the original author(s) and source are credited. 